\title{
Sexualité, prévention et rapports sociaux de sexe au fil de la vie
}

\author{
Nathalie BAJOs ${ }^{1}$, Michel Bozon², Nathalie BELTZER ${ }^{3}$ et l'équipe CSF $^{4}$
}

\section{Résumé}

Afin d'améliorer les capacités de négociation des femmes en matière de sexualité et de prévention, certains auteurs recommandent d'agir sur les représentations sociales qui opposent une sexualité féminine fondée sur l'affectivité et la conjugalité et une sexualité masculine qui s'appuierait sur des besoins physiques.

A partir de l'enquête Contexte de la sexualité en France (CSF), menée en 2006 auprès de 12364 personnes, cet article propose une lecture biographique des trajectoires sexuelles et préventives et une analyse des dimensions normatives les plus structurantes.

Les résultats attestent d'un rapprochement des pratiques sexuelles féminines et masculines mais également de la stabilité des représentations différentialistes de la sexualité (qui attribuent à la nature les différences entre femmes et hommes). Révélatrices des inégalités sociales de sexe qui prévalent dans d'autres sphères, notamment professionnelles et familiales, ces représentations génèrent des tensions qui rendent plus difficile pour les femmes l'adoption de pratiques préventives.

L'enjeurevientalorsàcréerlesconditionssocialesdel'égalitédespratiques concrètes entre les sexes dans les différentes sphères sociales.

Mots clefs : sexualité, genre, prévention, biographie, représentations sociales.

À la suite de la diffusion de l'épidémie à VIH dans les années 1980, les enquêtes sur les comportements sexuels se sont multipliées. Dans tous les pays où elles ont été menées, malgré des rapprochements dans les comportements sexuels des hommes et des femmes au fil des générations, on enregistre des différences systématiques de déclarations entre les uns et les autres, tant dans les pratiques que dans les représentations de la sexualité [1]. Si les âges aux premiers rapports sexuels se rapprochent [2] et les trajectoires affectives et sexuelles se diversifient, une dichotomie quasi universelle semble se maintenir dans les représentations sociales, opposant une sexualité féminine fondée sur l'affectivité, la conjugalité et la procréation, et une sexualité masculine

\footnotetext{
1 Unité Inserm 822/Ined, Santé reproductive, sexualité et infection à VIH, Le Kremlin Bicêtre. 2 Institut national d'études démographiques (Ined), Paris.

3 Observatoire régional de la santé d'île-de-France, Paris.

4 Équipe de l'enquête Contexte de la sexualité en France (CSF) : Nathalie Bajos et Michel Bozon (responsables scientifiques), Nathalie Beltzer (coordinatrice), Armelle Andro, Michèle Ferrand, Véronique Goulet, Anne Laporte, Charlotte Le Van, Henri Leridon, Sharman Levinson, Nicolas Razafindsima, Laurent Toulemon, Agnès Prudhomme, Josiane Warszawski.
}

qui s'appuierait sur des besoins physiques. Fonctionnant comme norme de comportement, cette dichotomie peut conduire les femmes à une plus grande vulnérabilité dans la négociation de pratiques préventives, en particulier lorsqu'elles vivent une sexualité qui ne s'inscrit pas dans un cadre conjugal [3].

Une analyse récente des actions de prévention du VIH a permis d'établir que les plus efficaces étaient celles qui visaientà modifier les déterminants sociaux des pratiques sexuelles et à risque, telles la pauvreté, les migrations, l'absence d'autonomie sociale et financière des femmes. Pour autant, nombre d'auteurs en appellent à modifier les représentations sociales en matière de sexualité, afin d'accroître les capacités de négociation des femmes [4].

Les données de l'enquête sur le contexte de la sexualité en France (CSF), menée en 2006 auprès d'un échantillon représentatif de la population vivant en France, permettent d'interroger cette perspective de modification des représentations. L'enquête explore finement les pratiques sexuelles, mais également les représentations de la sexualité, ainsi d'ailleurs que les représentations et les pratiques dans les sphères du travail, de l'éducation et de la vie domestique.

L'objectif de cet article est de proposer une lecture biographique des trajectoires sexuelles, pour donner à voir comment les différences entre hommes et femmes se construisent tout au long d'un parcours de vie, ainsi que la manière dont sont gérés les enjeux préventifs aux différentes étapes de ce parcours. Sont abordés le premier rapport sexuel, la vie affective et sexuelle entre ce premier rapport et la première mise en couple, les relations sexuelles après une rupture amoureuse et la sexualité aux âges avancés. Deux dimensions du contexte normatif de la sexualité sont analysées, la tendance à dissocier ou associer sexualité et affectivité d'une part, et l'adhésion à une vision biologique et naturalisante de la sexualité d'autre part, qui éclairent les différences entre sexes mises en évidence dans l'analyse des biographies sexuelles.

Notre analyse s'appuie sur les données de l'enquête sur le contexte de la sexualité en France (CSF) ${ }^{5}$ dont les premiers résultats viennent d'être publiés [5].

Cette enquête a été menée en 2006 à l'initiative de l'Agence nationale de recherches sur le sida et les hépatites virales (Anrs). Elle a été financée par l'Anrs, I'Inserm, I'Ined, la Fondation de France, la Direction de la recherche, des études, de l'évaluation et des statistiques (DREES) du ministère de la Santé, et I'Institut national pour la prévention en santé (Inpes). 


\section{Méthodologie de l'enquête sur le contexte de la sexualité en France}

L'enquête Contexte de la sexualité en France, réalisée en 2006 à l'initiative de l'Anrs, est la troisième enquête quantitative nationale sur les comportements sexuels en France, après celle de 1970 [21], et l'enquête Analyse des comportements sexuels en France (ACSF) de 1992 [22].

Son objectif est d'étudier le contexte social d'exercice de la sexualité en France, en appréhendant à la fois des actes, des relations et des représentations, en lien avec le contexte social, les conditions de vie, les trajectoires personnelles et les formes prises par les rapports entre les femmes et les hommes. L'objet de l'enquête est d'établir le lien entre sexualité et santé, aussi bien en ce qui concerne la protection contre le VIH/sida que la contraception, les interruptions volontaires de grossesse, les dysfonctionnements, les violences sexuelles, et les infections sexuellement transmissibles.

Comme l'enquête réalisée en 1992, il s'agit d'une enquête téléphonique, bien adaptée à un thème comme la sexualité. Elle a été menée auprès d'un échantillon aléatoire de la population âgée de 18 à 69 ans. Au total, 6824 femmes et 5540 hommes (soit 12364 personnes) ont été interrogés entre septembre 2005 et mars 2006, pour l'essentiel à travers des numéros de téléphone fixes, mais également à partir d'un échantillon complémentaire de téléphones cellulaires.

À la fin de l'enquête, une partie de l'échantillon s'est vu proposer un test de dépistage à domicile des chlamydiae, la chlamydiose étant l'infection sexuellement transmissible bactérienne la plus répandue en France.

Afin d'obtenir une probabilité égale d'être tiré au sort pour chaque individu, un coefficient de pondération égal à la taille du ménage a été appliqué. Ensuite, pour rendre la structure similaire à celle de la population française, l'échantillon a été redressé sur la base de l'enquête emploi de 2004 de l'Insee. Enfin, pour inclure dans l'échantillon général, les 304 personnes possédant uniquement un téléphone mobile, un poids proportionnel à leur effectif dans l'enquête leur a été attribué.

La participation à l'enquête s'est avérée particulièrement satisfaisante, puisque, au total, $74,6 \%$ des personnes sélectionnées ont accepté de répondre au questionnaire.

\section{L'entrée des femmes et des hommes dans la sexualité : un âge identique mais des expériences toujours spécifiques}

En un demi-siècle, l'âge médian des hommes au premier rapport sexuel s'est abaissé d'un an et demi (de 18,8 ans pour les générations âgées aujourd'hui de 65 à 69 ans, à 17,2 ans pour les jeunes de 18-19 ans), alors que celui des femmes, ini- tialement beaucoup plus élevé ( 20,6 ans), a chuté de trois ans (17,6 ans pour les femmes âgées de 18-19 ans). II en résulte un rapprochement des âges des hommes et des femmes au premier rapport [6]. Alors que chez les plus âgées, les femmes ont connu leur initiation sexuelle deux ans plus tard en moyenne que les hommes, il n'y a plus aujourd'hui que quelques mois de différence $(0,4$ an).

Cette similitude d'âge médian masque toutefois des calendriers d'entrée dans la sexualité qui restent différents pour les deux sexes : la proportion d'hommes de 18-29 ans ayant eu leur premier rapport avant l'âge de 15 ans est près du double de celle des femmes du même âge (25,3\% versus $14,2 \%)$. A l'inverse, les femmes sont plus nombreuses à déclarer un premier rapport « tardif » (32,1\% à 19 ans ou plus versus $23,1 \%$ pour les hommes). Mais surtout, en dépit de cette grande proximité des âges médians, cet événement continue à recevoir des lectures très différentes selon le sexe des individus. Certes, le temps de la découverte avant la sexualisation de la relation est le même pour les femmes et pour les hommes, environ six mois entre la première rencontre et le premier rapport, mais celui-ci ne se déroule pas dans le même contexte relationnel. Les rapports entre deux personnes non initiées ont augmenté au fil des générations mais ne sont toujours pas les plus fréquents : $31 \%$ d'après les déclarations des femmes de 18-29 ans, et $49 \%$ d'après celles des hommes du même âge. Les femmes ont plus souvent un premier rapport avec un partenaire déjà initié et plus âgé, voire beaucoup plus âgé qu'elles (tableau 1). Elles sont également plus nombreuses que leurs partenaires à déclarer qu'elles auraient préféré que ce premier rapport ait eu lieu plus tard (15,8\% versus $7,3 \%$ ). Leurs attentes se situent dans un autre registre que celles des hommes: pour elles, la logique affective l'emporte (43,3\% déclarent qu'elles ont eu ce premier rapport par amour et tendresse, $4,4 \%$ pour faire plaisir au partenaire), tandis que les hommes se réfèrent à un registre plus individuel, le désir ou la curiosité ( $43,7 \%$ et $15,2 \%$ ). D'ailleurs, les premières amours des jeunes femmes s'inscrivent dans un contexte relationnel plus stable que celles des jeunes hommes: pour deux tiers d'entre elles, la relation a duré plus de six mois tandis que ce n'est le cas que pour à peine un homme sur deux (tableau 1).

D'autres indicateurs attestent que le tout premier rapport sexuel est vécu bien différemment par les femmes et les hommes. Les femmes s'initient plus souvent que les hommes avec un partenaire sensiblement plus âgé. Elles sont plus préoccupées par les enjeux d'une grossesse non prévue $(23,4 \%$ contre $15,8 \%$ des hommes), bien qu'elles déclarent avoir utilisé un préservatif tout autant que leur partenaire et que $40 \%$ d'entre elles utilisaient la pilule ( $33 \%$ des jeunes femmes ont utilisé simultanément pilule et préservatif). Et si elles ont "logiquement " davantage abordé la question de la contraception avant le rapport, elles sont aussi plus nombreuses à avoir parlé des infections sexuellement transmissibles, qui les concernent tout autant que leurs 
Tableau 1

Caractéristiques et circonstances du premier rapport sexuel chez les personnes de 18 à 29 ans, selon le sexe

\begin{tabular}{|c|c|c|}
\hline & Femmes & Hommes \\
\hline $\begin{array}{l}\text { Âge du premier rapport } \\
\text { Âge médian (ans) } \\
\text { Initié(e)s à } 15 \text { ans ou moins } \\
\text { Initié(e)s à } 19 \text { ans ou plus } \\
\text { Initié(es) avec un partenaire non vierge }\end{array}$ & $\begin{array}{r}18,0 \text { ans } \\
14,2 \% \\
32,1 \% \\
68,3 \%\end{array}$ & $\begin{array}{r}17,4 \text { ans } \\
25,3 \% \\
23,1 \% \\
48,8 \%\end{array}$ \\
\hline $\begin{array}{l}\text { Motif principal du premier rapport } \\
\text { Amour, tendresse } \\
\text { Faire plaisir au partenaire } \\
\text { Désir } \\
\text { Curiosité } \\
\text { Franchir une étape } \\
\text { Pour faire comme les amis } \\
\text { Total }\end{array}$ & $\begin{array}{r}43,3 \% \\
4,4 \% \\
25,6 \% \\
11,7 \% \\
12,6 \% \\
1,3 \% \\
100\end{array}$ & $\begin{array}{r}20,2 \% \\
1,3 \% \\
43,7 \% \\
15,2 \% \\
16,3 \% \\
2,3 \% \\
100\end{array}$ \\
\hline $\begin{array}{l}\text { Caractéristiques de la relation } \\
\text { Durée médiane entre la rencontre et le premier rapport } \\
\text { Proportions de premiers partenaires du même âge (entre }-1 \text { an et }+1 \text { an) } \\
\text { Proportions de premiers partenaires âgés d'au moins } 5 \text { ans de plus } \\
\text { Devenir de la relation avec le premier partenaire } \\
\text { Seul rapport avec ce partenaire } \\
\text { - Durée de moins de } 6 \text { mois } \\
\text { - Durée de plus de } 6 \text { mois (pas vécu ensemble) } \\
\text { - Vivent ou ont vécu ensemble } \\
\text { - Total }\end{array}$ & $\begin{array}{r}6 \text { mois } \\
44,1 \% \\
18,5 \% \\
\\
16,0 \% \\
17,7 \% \\
33,6 \% \\
23,5 \% \\
100 \\
\end{array}$ & $\begin{array}{r}5,5 \text { mois } \\
74,2 \% \\
3,9 \% \\
\\
26,4 \% \\
26,5 \% \\
29,7 \% \\
11,0 \% \\
100\end{array}$ \\
\hline $\begin{array}{l}\text { Contraception - Prévention } \\
\text { Très peur à l'idée de tomber enceinte (ou à l'idée que la partenaire tombe enceinte) } \\
\text { A parlé de contraception et de sida avant ce premier rapport } \\
\text { - A parlé de contraception } \\
\text { - A parlé de MST*/sida } \\
\text { - N'a parlé ni de contraception ni de MST/sida } \\
\text { Préservatif et pilule } \\
\text { - Préservatif } \\
\text { - Pilule }\end{array}$ & $\begin{array}{l}23,4 \% \\
63,7 \% \\
44,7 \% \\
28,1 \% \\
\\
85,3 \% \\
39,6 \% \\
\end{array}$ & $\begin{array}{l}15,8 \% \\
48,0 \% \\
35,5 \% \\
42,4 \% \\
\\
83,7 \% \\
16,3 \% \\
\end{array}$ \\
\hline
\end{tabular}

Source : enquête CSF Inserm/Ined/Anrs 2006.

-Maladie sexuellement transmissible.

homologues masculins. La prise en charge des enjeux de santé sexuelle et reproductive relève donc encore de la responsabilité féminine dans les jeunes générations, et ce dès la phase d'initiation à la sexualité relationnelle, alors même que peu d'entre elles ont déjà entamé un suivi gynécologique pour les questions de contraception.

Si globalement, femmes et hommes déclarent dans des proportions remarquablement similaires et très élevées avoir utilisé un préservatif au premier rapport ( $85,3 \%$ des femmes et $83,7 \%$ des hommes de 20-29 ans), les circonstances d'entrée dans la sexualité ne sont pas sans effet sur les démarches contraceptives et préventives adoptées. Les femmes et les hommes qui s'initient avec un partenaire considéré comme le futur conjoint ont moins souvent utilisé un préservatif [7]. Cette moindre utilisation du préservatif n'est pas liée à un désir de grossesse mais sans doute au fait que le risque d'infection par le VIH semble écarté dans un tel contexte relationnel. De fait, la pilule seule est plus souvent utilisée dans le cadre de ces rapports : $11,4 \%$ contre 5,3\% quand le partenaire n'est pas considéré comme le futur conjoint. Par ailleurs, quand les femmes déclarent qu'elles n'avaient pas envie de ce rapport, un préservatif est moins souvent utilisé que lorsqu'elles déclarent qu'elles souhaitaient ce rapport. De même, lorsqu'elles s'initient avec un partenaire plus âgé, le préservatif est moins utilisé ; l'inverse ne se vérifie pas : si la femme est plus âgée que son partenaire, cela n'a pas d'incidence sur les comportements de prévention [7].

Les conditions de ce premier rapport constituent un bon élément de prédiction de ce qui va se jouer dans la sexualité adulte [6,8], ainsi que des attitudes et des pratiques à l'égard des risques d'infection sexuellement transmissible $[7,9,10]$. 


\section{Entre le premier rapport et la première vie en couple : quand les trajectoires féminines se différencient de celles des hommes}

Les biographies affectives et sexuelles des femmes et des hommes se sont diversifiées au cours de ces dernières décennies et le premier partenaire n'est plus que rarement le premier conjoint [11]. Cet allongement de la phase de sexualité préconjugale est observé dans de nombreux pays du Nord et du Sud [4]. Au cours de cette période, les répertoires sexuels s'élaborent et les enjeux de contraception et de prévention s'avèrent particulièrement importants. Dans la continuité de l'expérience du premier rapport sexuel, cette période de la vie se construit de manière spécifique pour les femmes et les hommes.

Alors qu'en 1970, $68 \%$ des femmes et $18 \%$ des hommes déclaraient n'avoir eu qu'un seul partenaire au cours de leur vie, en 1992 , elles et ils étaient respectivement $43 \%$ et $21 \%$, pour n'être plus que $34 \%$ et $16 \%$ en 2006. Dans le même temps, l'âge de la première mise en couple a tendu à s'accroître, passant de 21,8 ans pour les femmes et 23,9 ans pour les hommes de la génération 1945 à 23,2 ans et 26,1 ans pour ceux de la génération 1972 [12].

Si l'on examine la situation des jeunes de 25-29 ans, les femmes ont plus souvent connu une expérience de vie en couple que les hommes ( $81,3 \%$ versus $68,4 \%$ ) et celle-ci est intervenue dans un laps de temps plus court après le premier rapport que pour leurs homologues masculins (tableau 2). Deux tiers d'entre elles ont ainsi expérimenté la vie conjugale moins de cinq ans après leur premier rapport, alors que ce n'est le cas que d'un homme sur deux environ. Plus brève, cette période pré-conjugale féminine apparait également moins diversifiée du point de vue des expériences sexuelles. Pour une femme sur deux, la mise en couple a eu lieu avec le premier ou le deuxième partenaire tandis qu'un homme sur trois seulement se trouve dans cette situation. Ces derniers sont d'ailleurs beaucoup plus nombreux que les femmes du même âge à rapporter avoir eu au moins cinq partenaires au cours de ladite période ( $35,8 \%$ versus $13,2 \%)$. Les femmes qui ont eu au moins deux partenaires au cours de cette phase de sexualité préconjugale rapportent d'ailleurs moins de « relations sans lendemain " que leurs homologues masculins ( $62,6 \%$ versus $78,1 \%$ ).

Des différences de nombre de partenaires se dessinent donc dès le début des trajectoires sexuelles. Les écarts s'amplifient par la suite, comme en attestent les nombres de partenaires au cours de la vie rapportés à des âges plus avancés [13]. Certes, les femmes aujourd'hui rapportent plus de partenaires qu'en 1992 $(4,4$ versus 3,3$)$ tandis que les déclarations des hommes restent stables $(11,4$ versus 11,5$)$, mais les écarts entre sexes restent marqués.

Alors que les femmes et les hommes déclaraient avoir utilisé un préservatif au premier rapport dans des proportions similaires, les
Tableau 2

Intervalle de temps et nombre de partenaires entre le premier rapport et la première mise en couple pour les personnes de 25 à 29 ans ayant déjà vécu en couple (même si elles ne vivent plus en couple actuellement)

Femmes Hommes

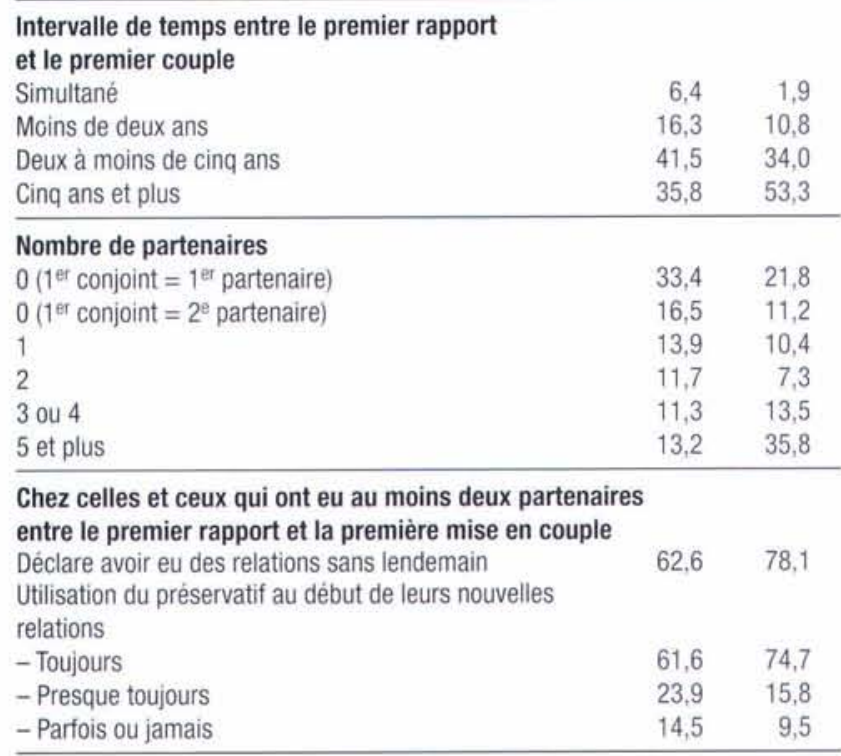

Source : enquête CSF Inserm/ined/Anrs 2006.

femmes rapportent moins souvent que les hommes avoir utilisé systématiquement un préservatif au début des nouvelles relations sexuelles qu'elles ont eu après leur première expérience. Certes, les partenaires des femmes de 25-29 ans sont pour certains plus âgés et appartiennent à des générations qui utilisent moins les préservatifs. Mais cet écart de déclaration peut également renvoyer aux difficultés auxquelles sont confrontées les femmes à négocier l'utilisation du préservatif dès lors qu'elles vivent une sexualité diversifiée, qui reste socialement moins acceptée pour elles que pour les hommes [14].

\section{Après une rupture : une vulnérabilité féminine plus marquée}

La diversification des biographies affectives et sexuelles au cours de ces dernières décennies s'accompagne de phases de rupture de plus en plus fréquentes. Celles qui surviennent avant l'âge de 25 ans concernent essentiellement des relations non conjugales dans lesquelles les enjeux de parentalité, effectifs ou envisagés, ne sont guère présents. Au-delà de cet âge, les femmes et les hommes rapportent dans des proportions similaires avoir vécu ce type d'expérience dans les cinq dernières années, qu'il s'agisse de ruptures de relations conjugales ou de rupture d'avec 
Tableau 3

Nouvelles relations après une rupture survenue dans les cinq dernières années et utilisation du préservatif

\begin{tabular}{|c|c|c|c|c|c|c|c|c|}
\hline & \multicolumn{4}{|c|}{$25-34$ ans } & \multicolumn{4}{|c|}{$35-54$ ans } \\
\hline & \multicolumn{2}{|c|}{ Rupture conjugale } & \multicolumn{2}{|c|}{ Rupture non conjugale } & \multicolumn{2}{|c|}{ Rupture conjugale } & \multicolumn{2}{|c|}{ Rupture non conjugale } \\
\hline & Femme & Homme & Femme & Homme & Femme & Homme & Femme & Homme \\
\hline \multicolumn{9}{|c|}{ A connu une rupture dans les 5 ans } \\
\hline Effectif & 223 & 167 & 187 & 178 & 245 & 175 & 110 & 74 \\
\hline$\%$ de oui & 12,2 & 14,4 & 12,7 & 17,0 & 8,4 & 8,6 & 4,2 & 4,2 \\
\hline Seuil de signification & \multicolumn{2}{|c|}{$p=0,005$} & \multicolumn{2}{|c|}{$p=0,005$} & \multicolumn{2}{|c|}{$p>0,5$} & \multicolumn{2}{|c|}{$p>0,5$} \\
\hline \multicolumn{9}{|c|}{ A eu au moins un nouveau partenaire après la rupture } \\
\hline Effectif & 223 & 167 & 187 & 178 & 245 & 175 & 110 & 74 \\
\hline$\%$ de oui & 76,7 & 71,2 & 69,0 & 66,7 & 62,6 & 74,2 & 62,8 & 73,8 \\
\hline Seuil de signification & \multicolumn{2}{|c|}{$p=0,3$} & \multicolumn{2}{|c|}{$p=0,7$} & \multicolumn{2}{|c|}{$p=0,03$} & \multicolumn{2}{|c|}{$p=0,2$} \\
\hline \multicolumn{9}{|c|}{ Utilisation du préservatif lors du premier rapport avec ce nouveau partenaire } \\
\hline Effectif & 168 & 124 & 135 & 128 & 157 & 130 & 68 & 57 \\
\hline$\%$ de oui & 79,0 & 77,1 & 77,6 & 81,6 & 44,8 & 61,5 & 40,9 & 74,9 \\
\hline Seuil de signification & \multicolumn{2}{|c|}{$p=0,7$} & \multicolumn{2}{|c|}{$p=0,5$} & \multicolumn{2}{|c|}{$p=0,02$} & \multicolumn{2}{|c|}{$p=0,001$} \\
\hline \multicolumn{9}{|c|}{ Ce premier partenaire avait-il lui même un autre partenaire? } \\
\hline Effectif & 168 & 124 & 135 & 128 & 157 & 131 & 68 & 57 \\
\hline$\%$ de oui/probable & 38,9 & 39,8 & 32 & 35,7 & 40 & 49,5 & 36,7 & 56,8 \\
\hline Seuil de signification & \multicolumn{2}{|c|}{$p=0,9$} & \multicolumn{2}{|c|}{$p=0,6$} & \multicolumn{2}{|c|}{$p=0,2$} & \multicolumn{2}{|c|}{$p=0,06$} \\
\hline \multicolumn{9}{|c|}{$\begin{array}{l}\text { Utilisation du préservatif avec ce partenaire lorsque } \\
\text { ce partenaire a d'autres partenaires au moment de ce rapport }\end{array}$} \\
\hline Effectif & 65 & 52 & 45 & 41 & 64 & 62 & 26 & 30 \\
\hline$\%$ de oui/probable & 82,5 & 86,2 & 73,6 & 93,4 & 52,8 & 67,3 & 41,4 & 80,0 \\
\hline Seuil de signification & \multicolumn{2}{|c|}{$p=0,6$} & \multicolumn{2}{|c|}{$p=0,008$} & \multicolumn{2}{|c|}{$p=0,2$} & \multicolumn{2}{|c|}{$p=0,02$} \\
\hline
\end{tabular}

Source : enquête CSF Inserm/Ined/Anrs 2006

un partenaire " qui a compté ». Seuls les hommes de 25-34 ans rapportent un peu plus souvent que les femmes du même âge avoir connu récemment une rupture non conjugale : $17,0 \%$ versus $12,7 \%$ (tableau 3 ).

Après une rupture, les femmes et les hommes retrouvent aussi souvent un partenaire, à l'exception des femmes de plus de 35 ans qui ont connu une rupture conjugale (tableau 3). Cette plus faible propension des femmes à s'engager dans une nouvelle relation n'est pas plus marquée pour celles qui ont des enfants que pour les autres [7]. Elle peut résulter d'une moindre disponibilité affective des femmes qui s'étaient fortement engagées dans leur relation conjugale, mais traduire également une diversité plus restreinte de leurs réseaux de relations et de leurs espaces de socialisation.

Le préservatif est plus souvent utilisé par les personnes de 25-34 ans que par celles de 35-54 ans lors du premier rapport avec un nouveau partenaire après la rupture. Les plus jeunes ont dans leur grande majorité débuté leur vie sexuelle à l'ère du sida et ont acquis des habitudes préventives dès leurs premiers rapports sexuels, dont on a montré par ailleurs qu'elles perduraient bien au-delà des phases d'initiation [7]. Si aucune différence n'est enregistrée entre les femmes et les hommes de 25-34 ans, en revanche les femmes de 35-54 ans déclarent moins souvent que les hommes du même âge avoir utilisé un préservatif avec leur nouveau partenaire, que celui-ci succède à une rupture conjugale ou non conjugale. La moindre propension des femmes de 3554 ans à se protéger peut alors traduire des enjeux relationnels particuliers : négocier l'utilisation d'un préservatif peut être difficile quand on en a peu l'expérience, mais peut également laisser penser à son partenaire que l'on a eu une vie sexuelle diversifiée, ce qui reste moins accepté socialement pour les femmes que pour les hommes [14]. À un âge où il est plus difficile pour une femme que pour un homme de retrouver un partenaire après une rupture conjugale, la priorité peut être donnée à l'engagement affectif plutôt qu'à une gestion " rationnelle " des risques liés à la survenue d'un rapport sexuel non protégé [15].

Tout se passe donc comme si les différences femmes/hommes qui se donnent à voir dès l'entrée dans la sexualité et qui s'amplifient par la suite se retrouvaient à toutes les phases du cycle de vie. Et ces différences persistent jusqu'à un âge avancé, même lorsque les enjeux de maternité, qui continuent à structurer fortement l'identité féminine mais aussi le rapport à la sexualité, ne sont plus présents dans la sexualité [14]. 
Tableau 4

Caractéristiques d'activité sexuelle chez les personnes de 50-69 ans

\begin{tabular}{|c|c|c|c|c|}
\hline & $\begin{array}{c}\text { Femmes } \\
50-59\end{array}$ & $\begin{array}{c}\text { Femmes } \\
60-69\end{array}$ & $\begin{array}{c}\text { Hommes } \\
50-59\end{array}$ & $\begin{array}{c}\text { Hommes } \\
60-69\end{array}$ \\
\hline \multicolumn{5}{|l|}{$\begin{array}{l}\text { Pensez vous qu'il y aura un moment où la sexualité ne vous intéressera } \\
\text { plus du tout (\%)? }\end{array}$} \\
\hline 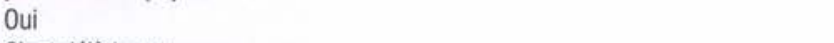 & 53,1 & 59,4 & 48,2 & 58,1 \\
\hline C'est déjà le cas & 3.9 & 14,8 & 1,5 & 3,5 \\
\hline Ne sait pas & 16,1 & 9,4 & 12,6 & 8,0 \\
\hline Non & 26,9 & 16,4 & 37,7 & 30,4 \\
\hline La sexualité est-elle indispensable à votre équilibre personnel ? (\% de oui) & 27,4 & 16,7 & 45,0 & 31,9 \\
\hline A au moins un partenaire actuellement (\%) & 80,6 & 62,6 & 86,8 & 84,0 \\
\hline A eu deux partenaires dans les 12 mois (\%) & 1,7 & 1,1 & 7.7 & 3,8 \\
\hline \multicolumn{4}{|l|}{ Absence ou insuffisance de désir sexuel pour celles et ceux qui ont un partenaire } & 42,5 \\
\hline \multicolumn{5}{|l|}{ Trouble de l'érection pour les hommes qui ont une partenaire } \\
\hline $\begin{array}{l}\text { Difficultés à atteindre l'orgasme pour les femmes qui ont un partenaire } \\
\text { (\% souvent, parfois) }\end{array}$ & 40,1 & 48,3 & - & - \\
\hline $\begin{array}{l}\text { Utilisation du Viagra au cours de la vie (hommes), utilisation de Viagra } \\
\text { par le partenaire (femmes) (\%) }\end{array}$ & 2,6 & 4,6 & 4,7 & 7,9 \\
\hline
\end{tabular}

Source : enquête CSF Inserm/Ined/Anrs 2006

\section{La sexualité aux âges avancés}

Un des traits marquants de l'évolution des comportements sexuels au cours de ces dernières décennies est le prolongement de l'activité sexuelle, surtout féminine, au-delà de la cinquantaine. Certes, les femmes de plus de 50 ans sont toujours moins nombreuses que les hommes du même âge à déclarer avoir un partenaire actuellement, ainsi qu'avoir eu deux partenaires dans les douze derniers mois (tableau 4), en raison notamment des plus grandes difficultés à trouver un partenaire (surmortalité masculine et préférence des hommes pour des partenaires plus jeunes). Mais lorsque les femmes de plus de 50 ans sont en couple, elles sont aujourd'hui près de $90 \%$ à déclarer une activité sexuelle dans les douze derniers mois, alors qu'elles n'étaient que $77 \%$ dans l'enquête Analyse des comportements sexuels en France de 1992, et $49 \%$ dans celle de 1970. La proportion des hommes en couple de plus de 50 ans qui ont une activité sexuelle s'élève également, mais beaucoup moins depuis les précédentes enquêtes (62 \% en 1970, $89 \%$ en 1992 et $95 \%$ en 2006). L'activité sexuelle des femmes les plus âgées continue ainsi à augmenter, tendance qui se dessinait dès le début des années 1970.

Si l'activité des femmes et des hommes de 50-69 ans apparaît plus proche aujourd'hui, le sens que les individus accordent à la sexualité reste, ici encore, différent pour les deux sexes. Le désintérêt pour la sexualité augmente avec l'âge, mais cette perte d'intérêt se manifeste plus tôt parmi les femmes. Par ailleurs, la sexualité est moins souvent perçue comme indispensable à l'équilibre personnel par les femmes, et ce surtout au-delà de 50 ans (tableau 4).

La prolongation de l'activité sexuelle à des âges plus avancés s'accompagne également de troubles de la sexualité de plus en plus courants, particulièrement chez les femmes. Elles rapportent plus fréquemment que les hommes avoir eu dans les douze derniers mois souvent ou parfois " une absence ou une insuffisance de désir sexuel ». On soulignera également qu'elles rapportent beaucoup plus de difficultés à atteindre l'orgasme que les hommes ne déclarent de troubles de l'érection. On peut penser qu'à troubles équivalents, les femmes déclarent plus facilement ceux$\mathrm{ci}$, une telle situation ne remettant pas aussi fondamentalement en cause leur identité sexuelle que pour les hommes. Si l'on examine le recours à des produits médicamenteux, comme le Viagra, pour favoriser l'érection masculine, l'augmentation avec l'âge est régulière. Les hommes de 50-59 ans déclarent 4,7\% d'utilisation, ceux de 60-69 ans 7,9\%. Les femmes de 50-59 ans déclarent $2,6 \%$ de recours au Viagra chez un de leurs partenaires, celles de 60-69 ans 4,6\%. En raison des écarts d'âge entre partenaires, on attendrait une utilisation plus élevée déclarée par les femmes : il y a donc visiblement un phénomène de dissimulation de la pratique par les hommes [6].

Les dérèglements physiologiques liés au vieillissement ne sauraient rendre compte de telles différences. Et I'on peut lire dans 
ces résultats le fruit d'une intériorisation de rôles sexuels tout au long d'un parcours de vie affective et sexuelle dans un contexte social qui reste, comme on va le voir, marqué par une dichotomie entre une sexualité féminine pensée sur le registre de l'affectivité et de la conjugalité et une sexualité masculine axée sur le désir et ses manifestations physiques.

\section{Divergences dans les représentations de la sexualité au féminin et au masculin}

Des transformations sensibles se dessinent au cours de ces dernières décennies dans les représentations de la sexualité, de plus en plus distinguée de ses enjeux de procréation. Toutefois, ces représentations continuent toujours à se conjuguer différemment au masculin et au féminin. L'évolution apparaît complexe, traduisant dans certains cas un rapprochement des positionnements des hommes et des femmes au fil des générations, dans d'autres un maintien, voire une accentuation des divergences entre les femmes et les hommes les plus jeunes.

Les femmes les plus âgées, qui déclarent, comme on l'a vu précédemment, une vie sexuelle plus diversifiée que leurs homologues des générations plus anciennes, restent toutefois peu nombreuses à envisager la sexualité en dehors du cadre d'une relation amoureuse. Les femmes de moins de 50 ans, qui ont débuté leur vie sexuelle alors que la contraception médicale s'était largement diffusée, dissocient à peine plus que leurs aînées les enjeux sexuels des enjeux affectifs et sont beaucoup moins nombreuses que les hommes à considérer "que l'on peut avoir des rapports sexuels avec quelqu'un sans l'aimer" (tableau 5). Alors qu'au fil des générations les hommes séparent de plus en plus sexualité et affectivité, les positions féminines évoluent peu, traduisant la pérennité de l'injonction sociale à une inscription de la sexualité dans une relation de couple. II en résulte que l'écart entre les déclarations des femmes et des hommes se creuse et une forte différence de point de vue est enregistrée chez les plus jeunes : les hommes de 18 à 24 ans sont deux fois plus nombreux que les femmes à considérer que l'on peut avoir des rapports sexuels avec quelqu'un sans l'aimer ( $57 \%$ contre $28 \%$ ). Seule, l'expérience d'une vie sexuelle diversifiée (plus de dix partenaires) semble autoriser une vision de la sexualité moins centrée sur l'affectivité, aussi bien pour les femmes que pour les hommes. En revanche, l'appartenance sociale des individus, qu'il s'agisse du niveau d'études, de la profession exercée, ou même de l'origine sociale (approchée par la profession exercée par les parents), n'influence pas l'adhésion à ce modèle largement dominant [14]. De même, les modes de vie (vivre en couple, avoir des enfants) ne modifient guère la manière de percevoir la relation entre affectivité et sexualité [14].

Ces divergences s'inscrivent dans une perspective différentialiste qui voitdanslabiologie lacause essentielle des différenceshommes/ femmes en matière de sexualité. Cette représentation prend souvent la forme d'une psychologie, qui se fonde sur le caractère supposé déterminant des différences biologiques. Ainsi, les femmes et, dans une moindre mesure, les hommes adhèrent majoritairementà l'idée selon laquelle "les hommes [auraient] par nature plus de besoins sexuels que les femmes " $75 \%$ des femmes et $62 \%$ des hommes). Cette idée l'emporte dans tous les groupes d'âge, et n'est un peu moins présente que chez les jeunes de 18 à 24 ans, parmi les femmes comme parmi les hommes (tableau 6). La distance à cette conception différentialiste de la sexualité dépend des capitaux culturels et sociaux : dans chaque génération, les femmes et les hommes les plus diplômés y adhèrent moins - ce qui rend d'ailleurs compte du fait que les jeunes générations sont moins différentialistes que leurs aînées puisque plus diplômées [14]. La parentalité est associée à une vision plus différentialiste, pour les femmes comme pour les hommes, alors que le fait de vivre ou non en couple ne l'est pas [14]. De tels résultats traduisent sans doute le fait que l'arrivée d'un enfant confirme et renforce l'idée d'une définition différente des rôles féminins et masculins. L'inscription de la maternité, mais pas de la paternité, dans le biologique et l'instinctuel justifie encore, dans les représentations comme dans la vie quotidienne, l'inégale répartition des tâches domestiques et parentales, qui résiste aux poussées égalitaires [16].

En revanche, l'adhésion à cette vision différentialiste dépend peu des expériences sexuelles des individus. Seuls, et presque paradoxalement, les hommes qui ont eu plus de 30 partenaires se démarquent en se déclarant moins convaincus de besoins sexuels masculins plus forts. La fréquence de l'activité sexuellen'a, quantà elle, aucune influence, ni pour les hommes ni pour les femmes [14]. Cette croyance n'est toutefois pas sans incidence sur les pratiques sexuelles concrètes, dans la mesure où les femmes qui considèrent que les hommes ont par nature plus de besoins sexuels qu'elles déclarent davantage " avoir eu des rapports sexuels pour faire plaisir à leur partenaire sans en avoir vraimentenvie "[14]. On peut lire dans ces résultats la difficulté pour un homme à énoncer une absence de désir sexuel, mais aussi, comme certains travaux l'ont déjà montré, l'idée que la naturalisation des besoins sexuels masculins établit leur caractère irrépressible [17]. Ce caractère irrépressible des besoins masculins justifie, pour les femmes, audelà de l'injonction symbolique de les assouvir [18], la contrainte sociale d'y céder $[19,20]$.

Ainsi, en dépit de certaines évolutions, les représentations de la sexualité restent marquées par un clivage qui continue d'opposer une sexualité féminine pensée prioritairement dans le registre de l'affectivité et de la conjugalité, à une sexualité masculine pensée majoritairement dans le registre des besoins naturels et du plaisir.

\section{La sexualité au cœur des rapports sociaux de sexe}

Les premiers résultats de l'enquête sur le contexte de la sexualité en France montrent que les différences en matière de sexualité 
Tableau 5

On peut avoir des rapports sexuels avec quelqu'un sans l'aimer

\begin{tabular}{|c|c|c|c|c|c|c|}
\hline & Tout à fait d'accord & Plutôt d'accord & Plutôt pas d'accord & Pas du tout d'accord & Total & $n$ \\
\hline \multicolumn{7}{|c|}{ Femmes } \\
\hline $18-24$ & 7,0 & 21,3 & 25,9 & 45,8 & 100 & 815 \\
\hline $25-34$ & 10,4 & 19,0 & 22,5 & 48,1 & 100 & 1687 \\
\hline $35-39$ & 10,5 & 16,2 & 21,8 & 51.4 & 100 & 1137 \\
\hline $40-49$ & 11,1 & 16,9 & 16,4 & 55,6 & 100 & 729 \\
\hline 50-59 & 8,1 & 14,1 & 21,6 & 56,2 & 100 & 735 \\
\hline $60-69$ & 8,7 & 12,0 & 14,4 & 64,9 & 100 & 623 \\
\hline Total & 9,4 & 16,6 & 20,3 & 53,8 & 100 & 5726 \\
\hline \multicolumn{7}{|c|}{ Hommes } \\
\hline $18-24$ & 19,9 & 36,6 & 19,2 & 24,2 & 100 & 732 \\
\hline $25-34$ & 21,7 & 31,9 & 20,2 & 26,2 & 100 & 1370 \\
\hline $35-39$ & 21,5 & 28,4 & 20,3 & 29,8 & 100 & 943 \\
\hline $40-49$ & 20,2 & 27,5 & 21,1 & 31,1 & 100 & 618 \\
\hline $50-59$ & 20,0 & 25,8 & 21,7 & 32,6 & 100 & 532 \\
\hline $60-69$ & 19,5 & 20,3 & 19,6 & 40,6 & 100 & 415 \\
\hline Total & 20,5 & 28,4 & 20,5 & 30,6 & 100 & 4610 \\
\hline
\end{tabular}

Source : enquête CSF Inserm/lned/Anrs 2006

Tableau 6

Par nature, les hommes ont plus de besoins sexuels que les femmes

\begin{tabular}{|c|c|c|c|c|c|c|c|}
\hline & $\begin{array}{l}\text { Tout à fait } \\
\text { d'accord }\end{array}$ & $\begin{array}{c}\text { Plutôt } \\
\text { d'accord }\end{array}$ & $\begin{array}{l}\text { Plutôt pas } \\
\text { d'accord }\end{array}$ & $\begin{array}{l}\text { Pas du tout } \\
\text { d'accord }\end{array}$ & Ne sait pas & Total & $\mathrm{n}$ \\
\hline \multicolumn{8}{|c|}{ Femmes } \\
\hline $18-24$ & 27,1 & 38,7 & 18,7 & 14,2 & 1,3 & 100 & 985 \\
\hline $25-34$ & 35,8 & 37,2 & 14,2 & 11,5 & 1,2 & 100 & 2005 \\
\hline $35-39$ & 40,2 & 36,7 & 11,2 & 10,4 & 1,5 & 100 & 1346 \\
\hline $40-49$ & 37,7 & 34,3 & 11,5 & 13 & 3,4 & 100 & 866 \\
\hline $50-59$ & 35,5 & 38,7 & 12,1 & 9,8 & 3,8 & 100 & 884 \\
\hline $60-69$ & 33,8 & 40,0 & 9,9 & 9,3 & 7,0 & 100 & 738 \\
\hline Total & 35,3 & 37,4 & 12,8 & 11,4 & 3,1 & 100 & 6824 \\
\hline \multicolumn{8}{|c|}{ Hommes } \\
\hline $18-24$ & 16,5 & 37,1 & 23,9 & 20,7 & 1,8 & 100 & 887 \\
\hline $25-34$ & 24,7 & 34,4 & 18,7 & 18,9 & 3,3 & 100 & 1640 \\
\hline $35-39$ & 29,6 & 33,8 & 17,5 & 15,9 & 3,2 & 100 & 1126 \\
\hline $40-49$ & 26,8 & 37,4 & 16,5 & 15,6 & 3,6 & 100 & 744 \\
\hline $50-59$ & 20,8 & 36,5 & 18,2 & 18,5 & 6,0 & 100 & 641 \\
\hline $60-69$ & 22,7 & 31,8 & 16,0 & 20,9 & 8,6 & 100 & 502 \\
\hline Total & 23,5 & 35,4 & 18,3 & 18,3 & 4,4 & 100 & 5540 \\
\hline
\end{tabular}

Source : enquête CSF Inserm/Ined/Anrs 2006 
entre femmes et hommes se construisent dès l'entrée dans la sexualité et tout au long des trajectoires affectives et sexuelles. Elles s'inscrivent, tout autant qu'elles prennent sens, dans un contexte normatif qui se structure toujours autour d'un clivage pensé comme naturel "sexualité féminine affective/besoins sexuels masculins ". Cette répartition des rôles sociaux sexués n'est pas sans effet sur les pratiques préventives et contribue à placer les femmes dans une situation de plus grande vulnérabilité sociale vis-à-vis de la négociation en matière de prévention.

Si nombre d'indicateurs attestent d'une évolution sensible des pratiques sexuelles des femmes (âge au premier rapport, nombre de partenaires, activité sexuelle après 50 ans), tandis que celles des hommes évoluent peu depuis quelques décennies, aboutissant de fait à un rapprochement des pratiques féminines et masculines, on observe dans le même temps que les représentations sociales de la sexualité évoluent peu. Ces résultats apportent un éclairage nouveau sur les tensions qui peuvent exister entre représentations et pratiques sociales.

Une autre perspective peut ainsi être adoptée, dans la lignée des approches anthropologiques, qui consiste à appréhender les représentations différentielles des sexualités féminines et masculines comme un révélateur des inégalités sociales de sexe dans les autres sphères sociales. Si la société française se caractérise par un arsenal juridique très égalitaire, il reste que la sphère politique, celle du travail et celle de la vie domestique sont toujours traversées concrètement par des inégalités entre femmes et hommes [16]. Les analyses de l'enquête montrent clairement que les femmes adhèrent d'autant plus à une vision différentialiste de la sexualité que celles-ci se trouvent en situation de tension normative, c'est-à-dire lorsque leurs représentations égalitaires relatives au travail et au partage des tâches se heurtent à des pratiques inégalitaires [14]. La référence très majoritaire à la reconnaissance de besoins sexuels masculins plus importants, alors même que l'égalité entre les sexes est considérée comme une aspiration légitime dans les autres sphères de la vie sociale, peut paraître paradoxale, sauf si l'on considère qu'elle permet d'atténuer les tensions entre l'affirmation d'aspirations égalitaires et les pratiques concrètes marquées par de fortes discriminations entre les sexes [14].

Dans cette perspective, l'enjeu demeure de créer les conditions sociales de l'égalité des pratiques concrètes entre les sexes dans les différentes sphères sociales. Les politiques préventives qui visent à modifier prioritairement les représentations sociales de la sexualité afin de favoriser l'empowerment des femmes dans la seule sphère de la sexualité et de la reproduction rencontrent ici leurs limites.

\section{Références bibliographiques}

1. Hubert M, Bajos N. Sandfort T, Eds. Sexual behaviour and HIV/AIDS in Europe : comparisons of national surveys. Social Aspects of AIDS. Londres : Taylor \& Francis, 1998, $442 \mathrm{p}$.

2. Bozon M. A quel âge les femmes et les hommes commencent-ils leur vie sexuelle? Comparaisons mondiales et évolutions récentes. Population et Sociétés $2003 ; 391,4$ p.

3. Bajos N. Social factors and the process of risk construction in HIV sexual transmission. Aids Care $1997 ; 9(2): 227$.

4. Wellings K, Collumbien M, Slaymaker E, Singh S, Hodges Z, Patel D, Bajos N. Sexual Behaviour in Context : A Global Perspective. Lancet $2006 ; 368(9548)$ : 1706-28.

5. Bajos N, Bozon M, Eds. L'enquête sur la sexualité en France. Pratiques, genre et santé. Paris : La Découverte, 2008.

6. Bozon M. Premier rapport sexuel, première relation : des passages attendus. In L'enquête sur la sexualité en France. Pratiques, genre et santé, N. Bajos, M. Bozon, Eds. Paris : La Découverte, 2008.

7. Beltzer N, Bajos N. De la contraception à la prévention: les enjeux de la négociation aux différentes étapes des trajectoires affectives et sexuelle. In L'enquête sur la sexualité en France. Pratiques, genre et santé, N Bajos, M. Bozon, Eds. Paris : La Découverte, 2008.

8. Bozon M. L'entrée dans la sexualité adulte. Le premier rapport et ses suites. Du calendrier aux attitudes. Population $1993 ; 5: 1317-1352$

9. Lagrange $\mathrm{H}$, Lhomond $\mathrm{B}$ et al. L'entrée dans la sexualité : les comportements des jeunes dans le contexte du sida. Paris : La Découverte, 1997, 431 p.

10. Bajos N, Ducot B, Spencer B, Spira A. ACSF group. Sexual risk taking, sociosexual biographies and sexual interaction: Elements of the french national survey on sexual behaviour. Social Science and Medicine $1997 ; 44(1): 25-40$.

11. Bozon M, Héran F. La formation du couple. Paris : La Découverte, collection Grands repères classiques, 2006, Paris.

12. Prioux F. L'âge à la première union en France : une évolution en deux temps. In Histoires de familles, histoires familiales. Les résultats de l'enquête Famille de 1999, C. Lefèvre et A. Filhon, Eds. Paris : Ined, 2005, p 201-222.

13. Leridon $\mathrm{H}$. Le nombre de partenaires: un certain rapprochement entre les femmes et les hommes, mais des comportements encore très différents. In L'enquête sur la sexualité en France. Pratiques, genre et santé, N Bajos, M Bozon, Eds. Paris : La Découverte, 2008.

14. Bajos $N$, Ferrand $M$, Andro $A$. La sexualité à l'épreuve de l'égalité. In L'enquête sur la sexualité en France. Pratiques, genre et santé, N Bajos, M Bozon, Eds. Paris : La Découverte, 2008.

15. Peto D, Remy J, Van Campenhoudt L, Hubert M. Sida : I'amour face à la peur. Paris : Lharmattan, collection Logiques sociales, 1992, 221 p.

16. Ferrand M. Féminin Masculin. Paris: La Découverte, collection Repères, 2004.

17. Héritier F. Masculin/Féminin, La pensée de la différence. Paris : Odile Jacob, 1996.

18. Bourdieu P. La Domination masculine. Paris : Seuil, 1998.

19. Tabet $P$. Fertilité naturelle, procréation forcée. In L'araisonnement des femmes, NC Mathieu, Ed. Paris : Éditions de l'EHESS, collection Cahiers de I'homme, 1985, p. 61-146.

20. Jaspard M. Sociologie des comportements sexuels. Paris : La Découverte, collection Repères, 2005, 122 p.

21. Simon P, Gondonneau J, Miromer L, Dourlen-Rollier AM. Rapport sur le comportement sexuel des français. Paris : R Julliard et P Charron, 1972.

22. Spira A, Bajos $\mathrm{N}$ et le groupe ACSF. Les comportements sexuels en France. Paris : La Documentation française, 1993. 